\title{
BMJ Open GAMBLINGLESS: FOR LIFE study protocol: a pragmatic randomised trial of an online cognitive-behavioural programme for disordered gambling
}

\author{
S S Merkouris, ${ }^{1}$ S N Rodda, ${ }^{1,2,3,4}$ D Austin, ${ }^{1}$ D I Lubman, ${ }^{2,3}$ P Harvey, ${ }^{5,6}$ \\ M Battersby, ${ }^{5} \mathrm{~J}$ Cunningham, ${ }^{7,8,9,10}$ T Lavis, ${ }^{5} \mathrm{D}$ Smith, ${ }^{5} \mathrm{~N}$ A Dowling ${ }^{1,11}$
}

To cite: Merkouris SS, Rodda SN, Austin D, et al. GAMBLINGLESS: FOR LIFE study protocol: a pragmatic randomised trial of an online cognitive-behavioural programme for disordered gambling. BMJ Open 2017;7: e014226. doi:10.1136/ bmjopen-2016-014226

- Prepublication history for this paper is available online To view these files please visit the journal online (http://dx.doi.org/10.1136/ bmjopen-2016-014226).

Received 9 September 2016 Revised 28 November 2016 Accepted 21 December 2016

\section{CrossMark}

For numbered affiliations see end of article.

Correspondence to

S S Merkouris:

stephanie.merkouris@deakin. edu.au

\section{ABSTRACT}

Introduction: The prevalence of disordered gambling worldwide has been estimated at $2.3 \%$. Only a small minority of disordered gamblers seek specialist face-toface treatment, and so a need for alternative treatment delivery models that capitalise on advances in communication technology, and use self-directed activity that can complement existing services has been identified. As such, the primary aim of this study is to evaluate an online self-directed cognitive-behavioural programme for disordered gambling (GamBLINGLESS: For LIFE).

Methods and analysis: The study will be a 2-arm, parallel group, pragmatic randomised trial. Participants will be randomly allocated to a pure self-directed (PSD) or guided self-directed (GSD) intervention. Participants in both groups will be asked to work through the 4 modules of the GamBLINGLESS programme over 8 weeks. Participants in the GSD intervention will also receive weekly emails of guidance and support from a gambling counsellor. A total of 200 participants will be recruited. Participants will be eligible if they reside in Australia, are aged 18 years and over, have access to the internet, have adequate knowledge of the English language, are seeking help for their own gambling problems and are willing to take part in the intervention and associated assessments. Assessments will be conducted at preintervention, and at 2, 3 and 12 months from preintervention. The primary outcome is gambling severity, assessed using the Gambling Symptom Assessment Scale. Secondary outcomes include gambling frequency, gambling expenditure, psychological distress, quality of life and additional help-seeking. Qualitative interviews will also be conducted with a subsample of participants and the Guides (counsellors).

Ethics and dissemination: The study has been approved by the Deakin University Human Research and Eastern Health Human Research Ethics Committees. Findings will be disseminated via report, peer-reviewed publications and conference presentations.

Trial registration number: ACTRN12615000864527; results.

\section{INTRODUCTION}

Gambling disorder is defined in the Diagnostic and Statistical Manual of Mental

\section{Strengths and limitations of this study}

- This is the first Australian study to examine the effectiveness of a guided online self-directed cognitive-behavioural programme for disordered gambling, which will help address the gap in available treatment options for disordered gambling.

- The use of gambling counsellors as guides, in combination with the pragmatic trial design and adjunctive qualitative methodology, will enable the translation of this research in to clinical practice and also allow for upskilling of current gambling clinicians.

- A potential limitation of this study, as with any online programme, is low engagement and follow-up rates.

Disorders (DSM-5) as problematic gambling behaviour that is persistent and recurring, and leads to substantial impairment and disruption to personal, family or vocational pursuits. ${ }^{1}$ Although disordered gambling prevalence rates vary across jurisdictions, standardised international disordered gambling prevalence rates have been estimated at 2.3\%. ${ }^{2}$ In Australia, national estimates identify rates of $0.4-0.6 \%$ for problem gambling, $1.9-3.7 \%$ for moderate-risk gambling and $3.0-7.7 \%$ for low-risk gambling. ${ }^{34}$ The consequences of disordered gambling are farreaching and include financial, legal and occupational difficulties, family and relationship breakdown, and intimate partner violence. ${ }^{5}{ }^{6}$ Furthermore, disordered gambling is highly comorbid with mental health disorders, such as mood, anxiety, alcohol and drug use disorders, impulse control disorders and personality disorders. ${ }^{78}$

While a diverse range of treatment options for disordered gambling are currently available, recent systematic reviews have indicated that cognitive-behavioural therapy (CBT) 
and motivational interviewing (MI) are efficacious in treating disordered gambling. ${ }^{9-12}$ These reviews have primarily focused on face-to-face delivery of these interventions, as this has been the dominant funded model of treatment to date. Evidence, however, indicates that only a small proportion of disordered gamblers $(8-17 \%$ in Australia ${ }^{13}$ ) access specialist face-to-face gambling services, suggesting that this mode of treatment delivery does not provide sufficient access to evidence-based treatment. ${ }^{14}$ Barriers to accessing face-to-face treatment include personal factors, such as shame and denial and resource limitations, such as the limited availability of trained clinicians, time requirements, treatment costs, scheduling conflicts, childcare requirements and geographic inaccessibility. ${ }^{15}$ There is therefore a need to examine the use of alternative treatment delivery models that capitalise on advances in technology, employ nontraditional service providers and involve self-directed interventions that can complement existing services. ${ }^{16}$

Traditionally, self-directed materials have been administered in the form of written self-directed workbooks. Increasingly, however, self-directed materials are being delivered via internet-based technologies. Self-directed and internet-based interventions have several advantages when compared with traditional face-to-face therapies. They are typically shorter and more cost-effective, facilitate immediate treatment and may be able to reach more people, particularly subpopulations where inequities in services exist. ${ }^{14} 1718$ Moreover, the lack of interpersonal contact and sense of anonymity involved in these interventions may attract people who are reluctant to attend traditional inperson services. ${ }^{19} 20$ Online interventions have also been found to be non-stigmatising and empowering interventions that allow people to engage in treatment at their own time and pace. ${ }^{21}$

Self-directed interventions can range from purely selfdirected strategies involving no professional guidance (pure self-directed (PSD), self-guided or unguided selfdirected) to treatments involving minimal support from a healthcare professional (guided self-directed $(\mathrm{GSD})) .{ }^{14}{ }^{22}$ The support provided in GSD interventions is typically supportive or facilitative in nature, with the aim of actively guiding patients in the use of a selfdirected protocol. ${ }^{23}$ Generally, guides do not deliver therapeutic content, but rather provide motivational support, monitor progress, clarify information contained within the self-directed protocol, review activities to ensure the correct application of techniques by the patients and address any technical questions or other issues that may arise. ${ }^{182325}$ GSD interventions are more readily disseminated than traditional therapies as they can be implemented by a wide range of mental health providers. ${ }^{14} 26$

PSD interventions have been shown to be an effective treatment for several disorders, including depression, anxiety disorders (ie, generalised anxiety disorder, panic disorder and social phobia) and bulimia nervosa, when compared with waitlist controls or treatment as usual. ${ }^{27-32}$
GSD interventions have also been shown to be efficient and effective independent treatments, with clinical guidelines recommending GSD for several disorders, including depression, anxiety, bulimia nervosa and binge eating disorder. $^{33}$ There is evidence that GSD interventions are more effective than treatment as usual or waiting list control groups $^{26} 3435$ and more effective than PSD interventions for these disorders. ${ }^{25} 28{ }^{34}$ Moreover, randomised controlled studies have shown that GSD as a sole treatment can be as effective as more intensive face-to-face therapies for several disorders, such as depression and anxiety. ${ }^{24} 343637$

Despite the evidence in other fields, there is a paucity of research investigating the effectiveness of self-directed interventions for the treatment of disordered gambling. One of the first studies in this area found that a selfdirected manual (with or without an assessment interview) reduced gambling behaviour at 3 and 6 months follow-up; however, an indepth assessment interview did not further improve outcomes. ${ }^{38}$ Several randomised controlled studies have since been conducted to examine the efficacy of a self-directed workbook combined with a single $20-45$ min MI therapy session, a selfdirected workbook only condition and a waitlist control condition. ${ }^{39}{ }^{40}$ An RCT by Hodgins $e t$ a $t^{40}$ demonstrated that the MI plus self-directed workbook condition produced better outcomes than the self-directed only and waitlist control conditions at one month follow-up. Although the differences between the two workbook conditions were not maintained at the 12-month follow-up evaluation, the MI plus self-directed workbook condition was found to be more effective than the workbook only condition at the 24-month follow-up. ${ }^{40} \mathrm{~A}$ subsequent study by Hodgins $e t a l^{39}$ revealed that the MI plus self-directed workbook condition was more effective than the self-directed workbook only and waitlist control conditions at post-treatment and 12 months follow-up; however, the addition of six telephone booster sessions in the MI and self-directed workbook group did not further improve outcomes. Similarly, Abbott $e t$ a $t^{42}$ compared the effectiveness of a standard telephone treatment, a single brief motivational interview, a single brief motivational interview plus self-directed workbook and a brief motivational interview plus workbook plus four booster MI sessions. Contrary to expectations, all treatment conditions produced comparable outcomes at the 12-month follow-up evaluation.

A randomised controlled trial (RCT), conducted by Petry et $a l^{43}$ compared a gamblers anonymous (GA) referral control condition with a GA referral plus CBT-based self-directed workbook condition and a GA referral plus therapist-delivered CBT condition. This study found that both CBT conditions (therapistdelivered and self-directed) produced better outcomes at post-treatment compared with the GA referral only condition. Finally, LaBrie $e t a t^{44}$ randomised participants to either a GSD toolkit, a self-directed toolkit or a waitlist control condition. The toolkit consisted of three sections 
based on a combination of inoculation, stage change and relapse prevention theory. In this study, guidance consisted of only one telephone call to discuss the toolkit at the beginning of treatment. This study found no difference between the self-directed toolkit and GSD toolkit interventions; however, both interventions reduced gambling abstinence compared with the waitlist control at the 3-month follow-up evaluation.

Despite their advantages, few studies have examined the use of online platforms for the delivery of selfdirected interventions for the treatment of disordered gambling. ${ }^{45}{ }^{46}$ Carlbring and $\mathrm{Smit}^{45}$ conducted an RCT involving an 8-week internet-based CBT programme with minimal therapist contact compared with a waitlist control. The internet-based programme involved four MI-based modules and four CBT-based modules. All modules contained information and exercises and ended with essay-style questions and, participants were required to post at least one message on an online discussion group for each module. The therapist contact involved emails on homework assignments and a weekly telephone call, lasting on average $15 \mathrm{~min}$, with the aim of providing positive feedback, encouragement and to respond to questions about the programme. Findings revealed that the internet-based programme resulted in significant improvement in gambling symptom severity, anxiety, depression and quality of life that was maintained up to 36 months post-treatment.

Casey $e t a t^{46}$ evaluated the effectiveness of an RCT involving internet-based CBT, internet-based CBT comprising the monitoring, feedback and support modules only (internet-MFS), and a waitlist control. Results found that the internet-based CBT programme resulted in significant improvements in gambling-related behaviour, depression, anxiety, stress and quality of life relative to the waitlist control group at post-treatment and that these therapeutic gains were maintained at the 12-month follow-up evaluation. In contrast, the internet-MFS group resulted in fewer improvements. This study also compared the effect sizes of the internet-based CBT programme and the delivery of the CBT programme delivered face-to-face by the same research team in an earlier study. The findings revealed that the face-to-face CBT programme was superior in improving gambling-related cognitions, but no differences were found between groups for gambling expenditure, gambling frequency, gambling urge and gambling refusal self-efficacy. Despite the limited evaluation of online self-directed programmes in the gambling field, this is an area of research that is growing. Currently, several ongoing trials are being conducted in Canada, including an RCT evaluating an online self-directed cognitive-behavioural and motivation therapy intervention for disordered gamblers, ${ }^{47}$ and an RCT evaluating an online intervention for disordered gamblers with comorbid mental health symptoms. ${ }^{48}$

Taken together, these studies provide mixed evidence for the efficacy of self-directed interventions for disordered gambling. Although the available studies have focused on PSD interventions, several of the intervention arms in these studies approximate GSD. These arms, however, generally involve the addition of motivational interviews to workbook only conditions, rather than adding guidance per se. Taken together, the findings suggest that PSD interventions ${ }^{43} 4446$ and interventions that approximate GSD ${ }^{39} 404445$ produce better outcomes than waitlist control groups in the treatment of disordered gambling. It remains uncertain, however, as to whether GSD interventions offer advantages over PSD interventions for disordered gamblers, with limited evidence suggesting that GSD-like interventions are more effective than PSD $^{39} 40$ or produce comparable outcomes. ${ }^{44}$

As such, further research is required to evaluate the effectiveness of self-directed interventions for disordered gambling, and specifically comparing the differential effectiveness of GSD and PSD interventions. Based on the limitations of the current evidence base, this research should examine the effectiveness of a GSD intervention comparable to that in other addiction and mental health fields, where guidance is defined as minimal therapist contact that is facilitative in nature, and consists of more than a single session. ${ }^{24}$ Although GSD interventions can be implemented by a wide range of treatment providers, research is required to determine the real-world applicability of such interventions. A pragmatic trial design will allow for the evaluation of these interventions in current services, and produce results that are generalisable and applicable in current services, through the use of current treatment providers serving as guides. ${ }^{49}$ In addition, the use of a qualitative methodology, which has been recommended as an important adjunct to any RCT, ${ }^{50}$ will allow for further exploration of the acceptability and feasibility of implementing GSD interventions in current gambling treatment services.

\section{AIMS AND HYPOTHESES}

The primary aim of this study is to investigate the effectiveness of an online self-directed cognitive-behavioural programme for gambling (GAMBLINGLess: For LiFE) on gambling symptom severity, gambling behaviours (frequency and expenditure), additional help-seeking, psychological distress and quality of life. A pragmatic randomised trial will be conducted, whereby the GamblingLess programme will be delivered under two different conditions: (1) PSD (without any practitioner guidance); this condition will serve as an active control condition, and (2) delivered with guidance delivered via email by practitioners from existing gambling treatment services (GSD). It is hypothesised that the GSD intervention will lead to better outcomes than the PSD intervention at the 2, 3 and 12-month follow-up evaluations, as assessed by reductions in gambling symptom severity, gambling behaviours and psychological distress, and increases in quality of life and help-seeking. 
Secondary aims are to: (1) explore the acceptability and feasibility of the GAMBLINGLEss programme by both users and current treatment providers; (2) identify the subgroups of problem gamblers who can most benefit from PSD and GSD programmes by identifying predictors of treatment engagement and outcomes; and (3) identify the processes or mechanisms that are responsible for changes in gambling outcomes following the self-directed intervention.

\section{METHODS}

\section{Trial design}

The study is a two-arm, parallel group, pragmatic randomised trial, with online follow-up evaluations conducted at 2, 3 and 12 months from preintervention. This trial has been registered with the Australian New Zealand Clinical Trials Registry (Trial ID: ACTRN12615000864527). The protocol manuscript is compliant with the SPIRIT statement. $^{51}$

\section{Recruitment and participants}

Participants will be recruited Australia-wide using numerous strategies. These will include advertisements in public places and health services (eg, general practitioner (GP) waiting rooms) and online advertisements through Facebook and Google (Google Adwords). Advertisements and links to the GamblingLess website will also be placed on various university and gambling-related websites (eg, Gambling Help Online, Victorian Responsible Gambling Foundation). Finally, counsellors from current gambling treatment services, including Victorian Gamblers Help Services, will assist with participant recruitment. Counsellors from the participating agencies will provide information about the GAMBLINGLESS programme to their clients who express an interest in seeking additional help for their own gambling problems.

Individuals will be eligible to participate in this trial if they: (1) currently reside in Australia; (2) express interest in seeking some type of help for their own gambling problems; (3) are 18 years of age or older; (4) have access to the internet; (5) have adequate knowledge of the English language; and (6) are willing to take part in the 8-week programme and complete brief assessment measures at 2, 3 and 12 months follow-up. Consistent with a typical pragmatic trial, this programme will be available to any interested individual, regardless of whether they are seeking other forms of assistance. ${ }^{52}$

\section{Procedure}

To register for the programme, individuals will be asked to provide an email address and password. At this stage, eligibility will be assessed by asking participants to confirm that they are 18 years of age or older and that they live in Australia. During this process, participants will be required to provide informed consent online. In order to consent, an automatic window will appear with a detailed study explanatory statement. Participants will be prompted to read this explanatory statement and will then be asked to agree to the terms and conditions if they wish to continue. On providing consent, participants are immediately directed to the online preintervention questionnaire, after which they are automatically randomised to one of the two interventions. Two, 3 and 12 months after completing the preintervention questionnaire, participants will be asked to complete an online follow-up questionnaire. See figure 1 for the study flow chart.

\section{Gamblingless: For Life programme}

The GamblingLess programme is an 8-week internetdelivered cognitive-behavioural self-directed programme designed to help people with gambling problems. The programme consists of four modules, with each module including 13-15 activities (see table 1). Each module takes 1-2 hours to complete. While it is recommended that participants complete all modules and activities in numerical order, the GAMBLingLess programme allows participants to complete as many activities as they like and in any order they choose. As one of the aims of this study is to explore the acceptability and feasibility of this comprehensive programme, with a view to developing a more refined brief programme in the future, it is not anticipated that participants will complete all activities in each module.

In Module 1-Getting Ready to Gamble Less, participants gain a better understanding and awareness of their gambling and how they may want it to change. This MI-based module starts with exercises relating to assessing participants gambling behaviour and exploring motivations and consequences of their gambling. Other exercises in this module have been designed to help

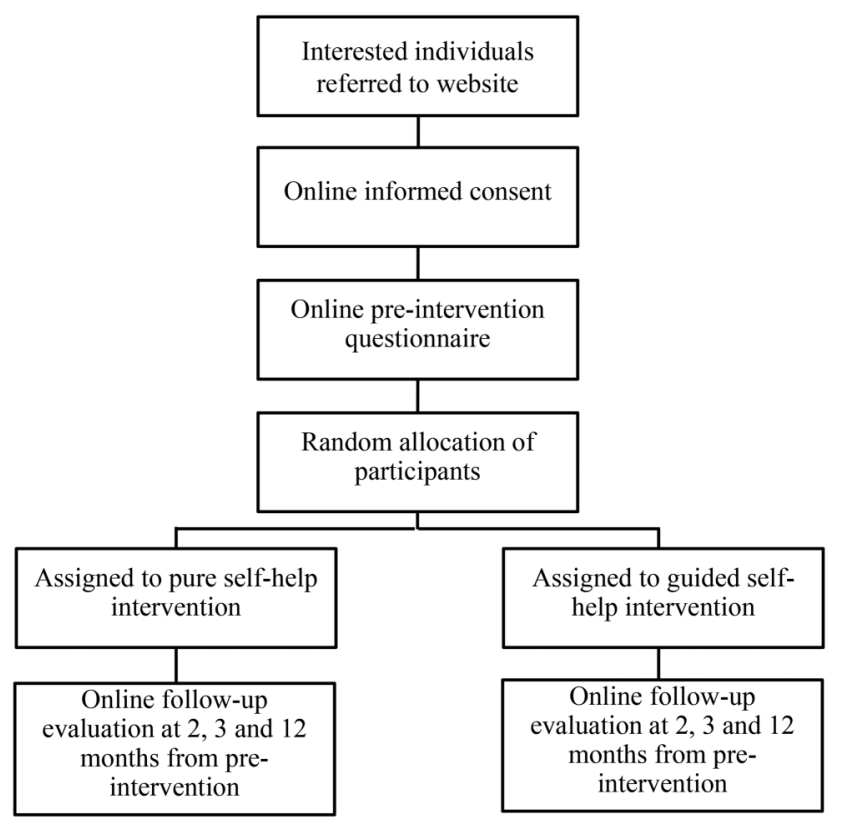

Figure 1 Participant flow chart. 
Table 1 Overview of the GamblingLess modules

\begin{tabular}{|c|c|c|c|}
\hline Module & Activities & Module & Activities \\
\hline \multirow{14}{*}{$\begin{array}{l}\text { 1. Getting ready } \\
\text { to gamble less }\end{array}$} & 1. Assessing my gambling & \multirow{14}{*}{$\begin{array}{l}\text { 2. Taking action to gamble } \\
\text { less }\end{array}$} & 1. My confidence to gamble less \\
\hline & 2. Understanding my gambling & & 2. Knowing my strengths \\
\hline & 3. My reasons for gambling & & $\begin{array}{l}\text { 3. My previous attempts to } \\
\text { gamble less }\end{array}$ \\
\hline & 4. My gambling triggers & & $\begin{array}{l}\text { 4. Limiting access to gambling } \\
\text { venues }\end{array}$ \\
\hline & $\begin{array}{l}\text { 5. My negative gambling } \\
\text { consequences }\end{array}$ & & 5. Guidelines to gamble safely \\
\hline & 6. Money I spend gambling & & 6. Limiting my access to money \\
\hline & 7. Keeping track of my gambling & & 7. My budget \\
\hline & $\begin{array}{l}\text { 8. Identifying the benefits of } \\
\text { gambling less }\end{array}$ & & 8. My enjoyable activities \\
\hline & 9. Knowing my values & & 9. Learning to relax I \\
\hline & 10. My future self & & 10. Learning to relax II \\
\hline & 11. My readiness to gamble less & & 11. Solving my problems I \\
\hline & 12. Deciding to quit or cut back & & 12. Solving my problems II \\
\hline & 13. Putting it all together & & 13. Seeking other help \\
\hline & Tracking my progress & & Tracking my progress \\
\hline \multirow[t]{16}{*}{$\begin{array}{l}\text { 3. Thinking differently } \\
\text { to gamble less }\end{array}$} & $\begin{array}{l}\text { 1. How my thoughts affect my } \\
\text { gambling }\end{array}$ & \multirow[t]{16}{*}{ 4. Gambling less for good } & $\begin{array}{l}\text { 1. Recognising my gambling } \\
\text { urges }\end{array}$ \\
\hline & 2. Adjusting my gambling thoughts & & 2. Managing my urges I \\
\hline & 3. The gamblers fallacy thinking trap & & 3. Managing my urges II \\
\hline & $\begin{array}{l}\text { 4. The illusion of control thinking } \\
\text { trap }\end{array}$ & & 4. Managing my urges III \\
\hline & 5. The prediction thinking trap & & 5. Managing my urges IV \\
\hline & 6. The chasing thinking trap & & $\begin{array}{l}\text { 6. Identifying my high-risk } \\
\text { situations }\end{array}$ \\
\hline & $\begin{array}{l}\text { 7. The positive expectancies } \\
\text { thinking trap }\end{array}$ & & $\begin{array}{l}\text { 7. Coping with my high-risk } \\
\text { situations }\end{array}$ \\
\hline & 8. The near miss thinking trap & & 8. The willpower breakdown \\
\hline & $\begin{array}{l}\text { 9. The low self-confidence thinking } \\
\text { trap }\end{array}$ & & $\begin{array}{l}\text { 9. My seemingly irrelevant } \\
\text { decisions }\end{array}$ \\
\hline & 10. The explanation thinking trap & & 10. My decision consequences \\
\hline & $\begin{array}{l}\text { 11. The selective memory thinking } \\
\text { trap }\end{array}$ & & 11. My reminder card \\
\hline & 12. Changing my thoughts I & & 12. Coping with my lapses \\
\hline & 13. Changing my thoughts II & & 13. My future \\
\hline & 14. Putting it all together I & & 14. Putting it all together \\
\hline & 15. Putting it all together II & & Tracking my progress \\
\hline & Tracking my progress & & \\
\hline
\end{tabular}

motivate participants, clearly outline the benefits of changing their gambling and understand their goals. In Module 2-Taking Action to Gamble Less, participants learn to identify skills and strategies they already use, and learn new skills, that will assist them in achieving their gambling goals. The strategies in this behavioural therapeutic module are designed to reduce and stabilise their gambling. Strategies include limiting access to gambling venues and money, budgeting, identifying alternative enjoyable activities, learning to relax, employing effective problem-solving strategies and seeking other help. Module 3-Thinking Differently to Gamble Less addresses common gambling-related maladaptive cognitions. In this cognitive therapeutic module, participants will learn how gambling works and some of the methods that the gambling industry uses to keep people gambling. This module has been designed to help participants make a more informed decision about their gambling. In doing so, participants will begin to understand which erroneous cognitions they are using and learn to modify them. Finally, Module 4-Gambling Less for Good helps increase participant awareness of the chain of events and behaviours leading up to a relapse, so that they can be more prepared to maintain their goals in the future. In this relapse prevention module, participants learn how to adapt some existing skills and learn some new skills to deal with high-risk situations and gambling urges so they do not relapse.

The development of the GaMBLINGLEss programme started with the collation of a comprehensive range of 
CBT and self-directed treatment manuals for disordered gambling. A consensus process by the Chief Investigators was conducted to identify the most relevant MI, cognitive and behavioural activities to develop a comprehensive and interactive protocol for the proposed intervention. Once the programme content was finalised by the Chief Investigators, it was adapted for online delivery. The online GamblingLess programme was then subjected to expert testing (a group of the Chief Investigators) and lay user testing (3 males and 1 female not involved in the development of the programme). The expert and lay user testing involved working through the entire programme, including the registration and consent process, the preintervention and module questionnaires, and all activities. Users were asked to provide feedback, using a structured feedback survey, on the content (eg, errors, level of difficulty, appropriateness of content), usability (eg, navigation, saving activities, rating each page) and likeability (eg, level of interest and engagement) of the programme. The feedback from the expert and lay user testing was collated and the online GamblingLess programme was refined accordingly. These refinements included editing or reducing the amount of text, adding interactive activities (eg, videos) and correcting minor technical issues.

The GamblingLess programme leverages the online delivery platform to engage participants through the use of interactive activities such as short videos, audio files, questionnaires and interactive animations. One of the unique features of the GamblingLess programme is that through the use of a save function, participants are able to save each activity as they complete it. This allows for a PDF version of the completed activities to be created at the end of the programme and therefore provides users with a permanent record of their treatment process and content. Another unique feature of the GamblingLess programme is that, throughout the 8-week intervention, participants are able to track their gambling spend, treatment goals and ability to resist gambling urges by completing a brief questionnaire administered automatically at the end of each module. Finally, an important feature of this programme is the built in rating item. At the end of each activity, participants will be asked to rate its helpfulness, using a five-star rating system. This enables the collection of important acceptability and feasibility data, while participants are using the programme.

\section{Interventions}

\section{Guided self-directed}

Participants allocated to the GSD intervention will be provided guidance throughout the 8-week programme via email. Consistent with the definition adopted in a recent meta-analysis, ${ }^{24}$ guidance will consist of a maximum of 1 contact per week (across the 8-week trial) with a maximum duration of 20 min per contact. Contacts will be via appointment-based email. They will be scheduled at the start of the intervention period, with guides initiating email contact once per week. Participants will be informed that they can email their guide as often as they like throughout the duration of the programme; however, the guide will only respond at the scheduled appointment time.

Guides will be responsible for providing assistance in a supportive and facilitative manner, with the aim of orienting the participant in the use of the GaMblingLess programme. The guides will provide support, monitor progress, clarify information contained within the programme, answer technical questions about internet delivery and address other problems that may arise. In these emails, participants will also be reminded to complete modules to enhance retention. As there is evidence to suggest that a clear deadline provided for the duration of the treatment can foster compliance, ${ }^{23} 53$ participants will be informed that they will only have access to the self-directed materials for 14 weeks and access to their guide for 8 weeks. The 8 -week guidance period coincides with the advice participants will receive to complete one module per fortnight. Participants who fail to attend their guidance appointments (ie, do not respond to the weekly email from their guides) will not be contacted by other means. Regardless of whether a participant responds, the guide will continue to send emails during the 8-week guidance period.

\section{Pure self-directed}

A PSD intervention will serve as an active control condition for this study. Participants in this group will have access to the same GamblingLess programme as participants in the GSD intervention; however, they will not receive weekly email support from a guide. Participants in the PSD intervention will also be informed that they will only have access to the GAMBLINGLEss programme for 14 weeks. An active control group was selected due to increasing awareness of the limitations of untreated control groups regarding ethical treatment and longterm follow-up of participants. Furthermore, given that GSD interventions are considered the gold standard in self-directed treatments for many disorders, with its efficacy and long-term efficiency proven in a sufficient number of RCTs, its superiority over an untreated control group does not seem to need further confirmation. $^{18}$

\section{Guide selection and training}

Approximately, 10 guides will be recruited from current gambling treatment services in Australia, namely, Gambling Help Online (the Australian national online counselling service) and Victorian Gamblers Help services. Secure project-specific email addresses will be provided to each guide. All emails exchanged between the guides and their participants will via these email addresses. The research team will collect the email exchanges between the Guides and their participants in 
secure servers at Deakin University and will assess them for treatment fidelity.

The GSD intervention will be manualised and the Guides will be required to complete a 3-hour training workshop. This workshop involves training the Guides in the use of the GamblingLess programme, how to communicate via email and how to provide guidance via email. Group peer supervision sessions will be conducted, with a member of the research team moderating these sessions. The supervision sessions will include discussions on the content of the email correspondence, a sharing of the experiences of providing guidance and discussions about problems encountered. In addition, the guides will have ongoing assistance and support from members of the research team, as required.

\section{Data collection}

Table 2 provides an overview of the evaluation timepoints at which each measure will be administered to participants throughout the trial. As mentioned previously, participants will be required to complete the preintervention questionnaire before gaining access to the GamblingLess programme. Participants will also be asked to complete module questionnaires within the programme and follow-up evaluations at 2, 3 and 12 months from completion of the preintervention questionnaire. Participants will be contacted via email to complete these follow-up evaluations. Participants will be compensated with an AUS $\$ 30$ gift voucher following the completion of each of the follow-up evaluations.

All questionnaires will be completed online. Short and modified versions of validated measures will be used to ensure that the online questionnaires are as brief as possible ( $15 \mathrm{~min})$. Across the evaluations, data were collected about sociodemographic information (eg, age, gender), gambling-related variables (eg, preferred gambling activity), psychosocial variables (eg, alcohol use), process measures (eg, gambling-related cognitions) and programme evaluation measures (eg, internet evaluation and utility questionnaire). At each evaluation time-point, data were collected on the primary outcome of gambling symptom severity, and secondary outcomes, including gambling behaviours, psychological distress, quality of life and additional help-seeking behaviour.

Participants who fail to complete a follow-up evaluation will receive two reminder emails. Participants who

Table 2 Overview of measures and time-points assessed

\begin{tabular}{|c|c|c|c|c|c|}
\hline Measure & Preintervention & $\begin{array}{l}\text { Module } \\
\text { questionnaire }\end{array}$ & 2-month follow-up & $\begin{array}{l}\text { 3-month } \\
\text { follow-up }\end{array}$ & $\begin{array}{l}\text { 12-month } \\
\text { follow-up }\end{array}$ \\
\hline \multicolumn{6}{|c|}{ Primary and secondary outcome measures } \\
\hline G-SAS & $x$ & $\begin{array}{l}\text { x (first } 4 \text { items } \\
\text { only) }\end{array}$ & G-SAS & $x$ & $\begin{array}{l}x \text { (first } 4 \text { items } \\
\text { only) }\end{array}$ \\
\hline Gambling behaviours & $x$ & $x$ & $\begin{array}{l}\text { Gambling } \\
\text { behaviours }\end{array}$ & $x$ & $x$ \\
\hline K6 & $\mathrm{x}$ & $\mathrm{x}$ & $\mathrm{K} 6$ & $\mathrm{x}$ & $x$ \\
\hline Quality of life & $\mathrm{x}$ & $\mathrm{x}$ & Quality of life & $\mathrm{x}$ & $\mathrm{x}$ \\
\hline $\begin{array}{l}\text { Additional help-seeking } \\
\text { behaviour }\end{array}$ & $x$ & $x$ & $\begin{array}{l}\text { Additional help } \\
\text { seeking behaviour }\end{array}$ & $x$ & $x$ \\
\hline $\begin{array}{l}\text { Sociodemographic } \\
\text { characteristics }\end{array}$ & $x$ & & & & \\
\hline \multicolumn{6}{|c|}{ Diagnostic and descriptive measures } \\
\hline PGSI & $x$ & & & & $\mathrm{x}$ \\
\hline Treatment goals & $\mathrm{x}$ & $x$ & $\mathrm{x}$ & $\mathrm{x}$ & $\mathrm{x}$ \\
\hline $\begin{array}{l}\text { Problematic gambling } \\
\text { activity }\end{array}$ & $\mathrm{x}$ & & & & \\
\hline Internet use & $\mathrm{x}$ & & & & \\
\hline Alcohol use & $\mathrm{x}$ & & $\mathrm{x}$ & $\mathrm{x}$ & $\mathrm{x}$ \\
\hline Substance use & $\mathrm{x}$ & & $\mathrm{x}$ & $\mathrm{x}$ & $\mathrm{x}$ \\
\hline \multicolumn{6}{|l|}{ Process measures } \\
\hline GRCS & $x$ & $x$ & $x$ & $x$ & $x$ \\
\hline AACRI & $\mathrm{x}$ & $\mathrm{x}$ & $\mathrm{x}$ & $\mathrm{x}$ & $\mathrm{x}$ \\
\hline Ready, Willing and Able & $\mathrm{x}$ & $x$ & $\mathrm{x}$ & $\mathrm{x}$ & $\mathrm{x}$ \\
\hline URICA-short form & $\mathrm{x}$ & & $\mathrm{x}$ & $\mathrm{x}$ & $\mathrm{x}$ \\
\hline Gambling BSCQ & $\mathrm{x}$ & & $\mathrm{x}$ & $\mathrm{x}$ & $\mathrm{x}$ \\
\hline \multicolumn{6}{|l|}{ Evaluation measures } \\
\hline $\begin{array}{l}\text { Internet evaluation and } \\
\text { Utility Questionnaire }\end{array}$ & & & $\mathrm{x}$ & & \\
\hline WAI-S & & & $\mathrm{x}$ & & \\
\hline $\begin{array}{l}\text { Additional requirements } \\
\text { from treatment }\end{array}$ & & & $x$ & & \\
\hline
\end{tabular}


fail to complete the follow-up evaluation after these two reminder emails will be contacted by a research assistant via telephone as a final reminder. Participants contacted over the telephone will be given the option of completing the follow-up evaluation with the assistance of the research assistant who will be blind to which treatment condition participants were allocated.

\section{Primary outcome}

\section{Gambling symptom severity}

The Gambling Symptom Assessment Scale (G-SAS) ${ }^{54}$ will be used to measure the severity of gambling symptoms. The G-SAS is a self-report scale that consists of 12 items designed to assess change in gambling symptom severity during treatment. The G-SAS uses a past week timeframe and each item is scored from 0 to 4 , with varying response options for each item. Total scores on the G-SAS range from 0 to 48, with higher scores indicating greater gambling symptom severity. Scores on the G-SAS can be categorised as extreme (41-48), severe (31-40), moderate (21-30) or mild (8-20). The first four items of the G-SAS can also be used as an assessment of change in gambling urges, with scores ranging from 0 to 16 . The G-SAS has demonstrated high internal consistency $(\alpha=0.87)$ and good convergent validity with other measures of gambling symptom severity. ${ }^{54}$

\section{Secondary outcomes}

\section{Gambling behaviours}

Past month gambling frequency will be assessed using self-report items relating to the number of days gambled on six types of gambling activities. These activities include electronic gaming machines (EGMs), table games (eg, blackjack), horse, harness or greyhound racing, sports and events betting, number games (eg, lotteries and bingo) and informal private games (eg, playing cards at home). Similarly, self-report items will be used to assess past month gambling expenditure on these gambling activities. Participants will be instructed to answer zero if they believe that they broke even or won money.

\section{Psychological distress}

Psychological distress will be measured using the Kessler 6 Psychological Distress Scale (K6) ${ }^{55}$ The K6 measures current and non-specific psychological distress in the past 4 weeks and comprises six items relating to the experience of specific symptoms of psychological distress, such as nervousness, agitation, psychological fatigue and depression. The response options for each item range from 0 (none of the time) to 4 (all of the time). Item scores are summed to obtain a total score between 0 and 24 and respondents are classified as being at low, moderate, high or very high risk. This measure has demonstrated high internal consistency and reliability $(\alpha=0.89)$ across major sociodemographic subsamples. ${ }^{55}$

\section{Quality of life}

The first item from the EUROHIS-QOL 8-item index will be used to assess overall quality of life ('How would you rate your quality of life?'). ${ }^{56}$ The EUROHIS-QOL 8 is a short version of the WHOQOL-BREF, the first item of which is the same across both measures. The response options for this item range from 1 (very poor) to 5 (very good). This item has been shown to be highly correlated with overall EUROHIS-QOL 8-item scores. ${ }^{56} 57$

\section{Additional help-seeking behaviour}

Participants will be asked to indicate how many times, if any, they have accessed other support options for gambling during the previous 30 days. The items relate to a range of support options, including talking to a gambling counsellor face-to-face or online via chat, calling a gambling helpline, sending an email to a gambling counsellor, reading or posting in gambling forums, attending support groups, speaking to family or friends, trying self-help strategies, seeking financial counselling, reading information or completing self-directed modules on the Gambling Help Online website, selfexcluding, signing up to residential gambling treatment or talking to a GP, psychologist or psychiatrist.

\section{Sociodemographics}

The following sociodemographic characteristics will be collected: age, gender, postcode of current residence, country of birth, employment status and personal net income per year.

\section{Diagnostic and descriptive measures \\ Problem gambling severity}

The Problem Gambling Severity Index (PGSI) ${ }^{58}$ will be used as a past year measure of problem gambling severity for diagnostic and sample descriptive purposes. The PGSI consists of 9 items rated on a 4-point Likert scale, ranging from 0 (never) to 3 (almost always). Scores range from 0 to 27 , whereby higher scores indicate greater problem severity. Scores on the PGSI can be used to classify individuals as non-problem gamblers (score of 0 ), low-risk gamblers (scores of 1 or 2), moderate-risk gamblers (scores between 3 and 7) or problem gamblers (scores of 8 or higher). The PGSI has high internal consistency $(\alpha=0.84)$ and demonstrates good criterion validity with measures of gambling involvement and gambling severity. ${ }^{58} 59$

\section{Treatment goals}

Participants' current goal for treatment will be examined, with the following response options provided: (1) quit (or stay quit) gambling altogether; (2) quit (or stay quit) the gambling activities I think I have an issue with; or (3) cut back (or stay cut back) the gambling activities I think I have an issue with. 


\section{Problematic gambling activity}

As a measure of problematic gambling activity, participants will be asked to indicate if they have an issue with a range of gambling activities, including EGMs, table games (eg, blackjack), horse, harness or greyhound racing, sports and events betting, number games (eg, lotteries and bingo) and informal private games (eg, playing cards at home).

\section{Internet use}

The frequency of internet use will be assessed using a one-item self-report measure. Participants will be asked to indicate, in a regular week, how many hours they would use the internet for work/personal/education/ recreation purposes.

\section{Alcohol use}

Alcohol use will be measured using the Alcohol Use Disorders Identification Test-3 (AUDIT-3). ${ }^{60}$ The AUDIT-3 uses the third item of the AUDIT, which measures the frequency of consumption of six or more drinks on one occasion. The response options range from 0 (never) to 4 (daily or almost daily). This item has shown to be an effective one-item screening measure for hazardous drinking with studies indicating adequate rates of sensitivity (0.79-0.89) and specificity (0.650.79 ), when using a cut-off score of 1 (ie, more than never), ${ }^{6061}$ against the 10-item AUDIT and measures of past-year heavy drinking.

\section{Substance use}

As a measure of substance use, participants will be asked about the frequency of illegal drug use or use of prescription medications for non-medical purposes, in the previous 30 days. $^{62}$ This single item has shown good sensitivity $(0.85-1.00)$ and specificity $(0.74-0.96)$ for detecting current drug problems or drug use disorders and self-report drug use, against the Composite International Diagnostic Interview (CIDI) Substance Abuse Module. ${ }^{62}$

\section{Process measures}

\section{Gambling-related cognitions}

Gambling-related cognitions will be measured using a series of single items from the Gambling-Related Cognitions Scale (GRCS). ${ }^{63}$ The GRCS consists of five subscales examining interpretative bias, illusion of control, predictive control, gambling-related expectancies and the perceived inability to stop gambling. Response options for each item range from 1 (strongly disagree) to 7 (strongly agree), with higher scores reflecting a higher level of erroneous gambling cognitions. The items employed in this evaluation will include one item from each subscale and will be selected as they best reflect the gambling-related cognitions addressed within the GamblingLess programme. These items have Cronbach's $\alpha$ coefficients ranging from 0.44 to $0.82 .{ }^{63}$

\section{Coping with gambling temptations}

Selected items from the Alcohol Abuse Coping Response Inventory (AACRI) ${ }^{64}$ will be adapted for gambling as a measure of participants' ability to cope with gambling temptations. Four items from the behavioural factor of the AACRI were selected. These items relate to reducing stress, avoiding situations and/or places where one used to gamble or leaving tempting situations, focusing on techniques that they know about how to gamble less, and to do other things when tempted. The response options for each item range from 0 (never) to 2 (always). These items will be selected as they are reflective of the activities within the GamblingLess programme.

\section{Readiness and confidence to change}

Ready, Willing and Able ${ }^{65}$ uses three readiness rulers to assess the importance, readiness and confidence of participants to limit or stop their gambling. Participants will be asked to indicate 'how important is it for you that you limit/stop your gambling', 'where does limiting/ stopping gambling fit on your list of priorities' and 'how confident are you that you could resist an urge to gamble'. Response options range from 1 to 10 , where higher scores indicate greater importance, readiness or confidence. Similar rulers have demonstrated good psychometric properties in other addictions ${ }^{66} 67$ and have been used in previous studies of treatment-seeking gamblers. ${ }^{65}$

\section{Readiness to change}

Selected items from the reduced drinking version of the University of Rhode Island Change Assessment (URICA), adapted for gambling, ${ }^{68}$ will be used to measure participants readiness to change. This version of the URICA consists of 12 items, 3 items for each precontemplation, contemplation, action and maintenance stage of change. The items are rated on a 5-point Likert scale from 1 (strongly disagree) to 5 (strongly agree). A single item from each subscale was selected that best represented the contents of the GamblingLess programme.

\section{Gambling-related self-efficacy}

The Brief Situational Confidence Questionnaire $(\mathrm{BSCQ})^{69}$ measures confidence in one's ability to resist drinking when faced with high-risk situations using a visual analogue scale where 0 equates to 'not at all confident' and 100 equates to 'totally confident'. The original BSCQ consists of eight items relating to unpleasant emotions, physical discomfort, pleasant emotions, testing control over drinking, urges and temptations, conflict with others, social pressures to drink and having pleasant times with others, and has demonstrated excellent internal consistency $(\alpha=0.85){ }^{69}$ The 8 -item BSCQ was adapted for gambling with two further items added. These additional items relate to participants confidence 
in resisting the urge to gamble when faced with situations involving financial pressures and alcohol or drugs.

\section{Programme evaluation measures}

Programme evaluation

The Internet Evaluation and Utility Questionnaire ${ }^{70} 71$ will be used as a measure of participants' experience and perceptions of the GamblingLess programme. This questionnaire consists of 15 items, with 13 of these items using a 5-point Likert scale ranging from 0 (not at all) to 4 (very). Higher scores on these items indicate good experiences and perceptions of the GamblingLess programme. These items assess ease of use, convenience, engagement, enjoyment, layout, privacy satisfaction, acceptability, usefulness, comprehension, credibility, likelihood of returning and mode of delivery. This questionnaire also includes two open-ended items relating to the most and least helpful parts of the GamblingLess programme. The Internet Evaluation and Utility Questionnaire has been shown to have adequate internal reliability $(\alpha=0.69) .{ }^{70}$

\section{Therapeutic alliance}

The Working Alliance Inventory -short form (WAI-S) ${ }^{72}$ is a 12-item measure of therapeutic alliance. Response options range from 1 (never) to 7 (always). The WAI-S consists of three subscales: the goal, task and bond subscales. The goal subscale assesses the agreement between a client and therapist on treatment goals, the task subscale examines the agreement between the client and therapist on how to achieve the goals, and the bond subscale examines the development of a personal bond between the client and therapist. ${ }^{72}$ The WAI-S total scores range from 7 to 84 with higher scores indicative of a stronger therapeutic relationship. The WAI-S has demonstrated excellent internal consistency $(\alpha=0.95){ }^{73}$ For the purpose of this trial, only participants who were allocated to the GSD intervention will complete the WAI-S as a measure of the working alliance between the guide and the participant.

\section{Additional requirements from treatment}

Participants will also be asked to indicate additional issues that they may still want help with after taking part in the programme. This questionnaire has been adapted from the What I Want For Treatment questionnaire. ${ }^{74}$ It includes 21 statements, scored on a 10-point Likert scale ranging from 1 (definitely no) to 10 (definitely yes), and includes a variety of issues, such as moodiness or depression, anger, alcohol, tobacco or substance use, legal issues and financial issues.

\section{Sample size}

Effect sizes between GSD and PSD interventions range from 0.34 to $0.67 .^{28} 34{ }^{75-77}$ A total sample size of 100 participants at postintervention will be needed to detect an effect size of 0.55 (Cohen's $d$ ) for the primary outcome with statistical power of $(1-\beta)=0.80$ in a two-tailed test $(\mathrm{p}<0.05)$. Follow-up attrition rates vary in RCTs of online interventions, with some studies indicating 3-month follow-up attrition rates as low as $8 \%$, and others as high as $89 \% .{ }^{78} 79$ Taking into account a conservative dropout rate of $50 \%$, we aim to recruit a sample of 200 participants.

\section{Randomisation}

Stratified block randomisation, using block sizes of 4 , will be used to randomly allocate participants. To ensure balance on potential confounders, randomisation will be stratified based on gender, median age and problem gambling severity using the PGSI (8+). The randomisation schedule will be applied via automated programming and will be monitored by a member of the research team.

\section{Statistical analyses}

An intention-to-treat approach will be used to assess the differential effectiveness of the GSD and PSD interventions. A 'per protocol' and 'as treated' analysis will also be conducted to assess the relative efficacy of GSD and PSD (ie, how well treatments work under perfect conditions) within a counterfactual framework. Inverse probability weighting will be used to reduce potential bias of treatment estimates. ${ }^{80}$

A generalised mixed-effects model approach will be used in the analysis of repeated measures for primary and secondary continuous and categorical outcomes. Mixed-effects models take into account the interindividual differences in intraindividual change with repeated responses and use all the available data on each participant. Outcome variables at baseline will be statistically adjusted when performing mixed-effects modelling. Furthermore, the randomisation process will take into account known confounders (eg, stratification variables) and unknown confounders (eg, additional helpseeking), and as such, the two interventions will be highly balanced. Mixed models are also unaffected by randomly missing data and therefore do not require imputation methods. Fixed effects in models will be intervention group (GSD or PSD), time in continuous form (intervention period and maintenance effects) and interaction between group and time. Random effects in the model will be at study participant level, and represent an upward or downward shift in the outcome measure from an overall regression line and rate of change over time. Linear and non-linear combinations of regression coefficients from mixed models will then be tested for treatment group effect at follow-up time points and estimated between-group mean differences will be presented along with CIs. Predicted estimates of treatment outcome at each time point will be calculated using fitted models of the data in order to examine patterns of individual change within each group. To interpret effect sizes and precision for ordinal and categorical outcomes, ORs and CIs will be calculated. 
To manage missing data, the trial will adhere to the following steps: (1) follow-up of all randomised individuals will be attempted, even if they withdraw from allocated therapy; (2) a relatively large timeframe will be allowed for each follow-up assessment and so response intervals and frequency of questionnaire completion will be expected to vary between individuals. Mixed models will be used to account for this unbalanced design and time will be entered into models as a continuous covariate from the date of first intervention (baseline) to date of each follow-up measurement; (3) the main analysis performed will be valid under a plausible assumption about the missing data and use all available data; (4) data will be collected on the ease of obtaining outcome data (eg, number of failed follow-up attempts) and used alongside sensitivity analyses to explore departures from the missing at random assumption. ${ }^{81}$

For secondary analyses, effect sizes presented as Cohen's d will be calculated for primary and secondary outcomes to provide an estimate of the magnitude of differences between groups and to allow comparisons with other published studies. ${ }^{82}$ Clinically significant change, as outlined by Jacobson and Truax, ${ }^{83}$ will also be measured for the GSAS and K6. At the final follow-up, each participant's status will be defined as 'recovered' (final score fell into the functional range and corresponded to a reliable change), 'improved' (final score corresponded to a reliable change, but fell into the dysfunctional range), 'unchanged' (final score did not correspond to a reliable change) or 'deteriorated' (final score corresponded to a reliable change in the negative direction). Where possible, a series of logistic regression models will be employed to determine which factors predict treatment outcome (ie, recovered/ improved cf. unchanged/deteriorated), treatment engagement (high engagement cf. low engagement) and follow-up attrition (non-completer of follow-up questionnaire cf. completer of follow-up questionnaire). A series of mixed-effects models will be employed to evaluate which variables moderate gambling outcomes for each treatment. Structural equation modelling or a series of hierarchical regression analyses will be used to identify which process measures mediate gambling outcomes.

\section{Qualitative studies}

\section{Participants' experiences of the GambLINGLESS programme}

Qualitative data will be collected from a subsample of the participants in the pragmatic trial. The aim of these qualitative interviews is to explore participants' experiences with, and to evaluate the acceptability of, the GamblingLess programme. Participants selected for inclusion will be broadly representative of the RCT study sample, according to age, gender and gambling severity. Only participants who, in the 2-month follow-up evaluation, agree to be contacted for a further indepth telephone interview will be approached.
These qualitative interviews will be semistructured and conducted by a clinically trained research fellow. The interview will focus on what participants saw as having changed over the course of therapy, their attributions of change and their perceptions of helpful and unhelpful aspects of the programme. Participants will be asked how helpful each module was in reducing gambling, what was most and least helpful, what was difficult and how each module may be improved. Approximately eight participants (four from the PSD intervention and four from the GSD intervention) will be interviewed. The interview will be conducted via telephone, after the 14-week access to the GamblingLess programme is terminated, and the 2 and 3-month follow-up evaluations have been conducted. The interview will be audio-recorded for transcription and data analysis purposes. Data will be analysed using thematic analysis, based on Braun and Clarke's ${ }^{84}$ guidelines for an inductive approach to datadriven coding.

\section{Guides experiences of the GamblingLess programme}

Qualitative data will also be collected from the guides delivering the GSD intervention. The aim of these qualitative interviews is to explore how the programme could be effectively integrated into clinical practice in existing gambling treatment services. All of the guides will be approached to be involved in the qualitative interview. These interviews will be semistructured and conducted by a clinically trained research fellow. Guides will be asked questions about the intervention, the client experience and clinical supervision. There will be an emphasis on how this intervention would fit in with their current practice and what recommendations they would have for integration of the programme into the gambling treatment service sector. All interviews will be audio-recorded and transcribed verbatim. Data will be analysed using thematic analysis via Braun and Clarke's ${ }^{84}$ guidelines for an inductive approach to datadriven coding.

\section{DISCUSSION}

\section{Strengths and limitations}

This is the first study in Australia to examine the effectiveness of a guided online self-directed intervention for disordered gambling. As most individuals with a gambling problem do not seek face-to-face treatment, this study may help address the gap in available treatment options for disordered gambling by evaluating an online CBT self-directed programme. Additionally, through the use of an online delivery platform, the GamblingLess programme has the potential to reach subgroups of people who would otherwise not receive, or have access to, psychological interventions, and therefore enabling greater access to a cost-effective treatment option for gambling. A further strength of this trial is the use of gambling counsellors as guides. This, in combination with the use of a pragmatic trial design alongside the 
use of an adjunctive qualitative methodology, will enable the translation of this research in to clinical practice and will allow for the upskilling of current gambling clinicians. Moreover, the usability and acceptability data obtained from the evaluation of this comprehensive programme will allow for the development of empirically supported brief online programmes for disordered gambling.

As with any online programme, potential limitations of this study are low programme engagement rates and low follow-up rates. The literature on internet-based psychological therapies indicates that dropout across all treatment stages (ie, pre-treatment, during treatment and follow-up) can be quite high, ranging from $2 \%$ to $83 \%{ }^{85}$ In an attempt to deal with potentially low follow-up rates, the data collection protocol will include multiple and varying modes of contact, including multiple attempts via email and telephone.

Another potential limitation of this study involves the impact of conducting qualitative interviews with participants, prior to the completion of all follow-up evaluations, on the main outcome results. While this does not affect the 2 and 3-month follow-up results, there are potential implications for the 12-month results. As such, additional analyses (eg, sensitivity analysis) will be conducted to ensure that participants, who took part in the qualitative interview, do not unduly effect the results at 12 months.

\section{Dissemination}

\section{Ethical and safety considerations}

Importantly, the contact details of emergency, crisis and other support services will be easily available to all individuals accessing the GamblingLess website (ie, before signing up to the programme) and throughout the GamblingLess programme. This list includes Australia-wide and state-specific emergency, crisis and support services. The list of emergency numbers and websites has been compiled from various reputable websites including suicide line, lifeline and gambling help online. In addition, suicidal ideation and/or attempts, in the previous 30 days, will be examined in the preintervention questionnaire using the first item from the Suicide Behaviors Questionnaire-Revised. ${ }^{86}$ While suicidal ideation or attempts is not an exclusion criterion for participation in this trial, participants who endorse any suicide ideation and/or attempts on this item will trigger a message advising them that this programme may not be best suited for them at this time and they should seek other forms of assistance that are more immediate and appropriate; this message will also include the contact details of appropriate emergency and crisis services.

\section{Data confidentiality}

The data will be stored on a secure server at the School of Psychology, Deakin University. An industry-standard encryption protocol will protect the confidential data.
The data will only be accessible by members of the research team. The guides will have access to very limited information about the specific participants allocated to them to facilitate their provision of guidance for this study. This includes the following information: age, gender, contact details (email address and telephone number, where provided), gambling expenditure, ability to resist gambling urges and treatment goals.

\section{Dissemination of findings}

The results of this trial will be submitted for publication in peer-reviewed journals and as a report to the funding body. Additionally, the results will be presented at conferences in Australia and Internationally.

\section{Author affiliations}

${ }^{1}$ School of Psychology, Deakin University, Geelong, Victoria, Australia

${ }^{2}$ Turning Point, Eastern Health, Fitzroy, Australia

${ }^{3}$ Eastern Health Clinical School, Monash University, Melbourne, Victoria, Australia

${ }^{4}$ Auckland University of Technology, Auckland, New Zealand

${ }^{5}$ School of Medicine, Flinders University, Adelaide, Australia

${ }^{6}$ School of Medicine, Deakin University, Geelong, Victoria, Australia

${ }^{7}$ Centre for Addiction and Mental Health, Toronto, Ontario, Canada

${ }^{8}$ Department of Psychiatry, University of Toronto, Toronto, Ontario, Canada

${ }^{9}$ Department of Psychology, University of Toronto, Toronto, Ontario, Canada

${ }^{10}$ Research School of Population Health, Australian National University,

Canberra, Australia

${ }^{11}$ Melbourne Graduate School of Education, University of Melbourne, Parkville, Australia

Acknowledgements The authors would like to acknowledge the work of the online resource developer, Eric 0.

Contributors NAD led the development of the intervention and design of the study with contributions from SNR, SSM, DIL, MB, PH, JC, DA, DS and TL. SSM led the drafting of the manuscript. All authors have read and approved the final manuscript.

Funding This study is funded by the Victorian Responsible Gambling Foundation.

Competing interests None declared.

Ethics approval Deakin University Human Research Ethics Committee (Ethics ID: 2014-123) and Eastern Health Human Research Ethics Committee (Ethics ID: E07/2015).

Provenance and peer review Not commissioned; externally peer reviewed.

Data sharing statement The trial is currently in the follow-up data collection phase.

Open Access This is an Open Access article distributed in accordance with the Creative Commons Attribution Non Commercial (CC BY-NC 4.0) license, which permits others to distribute, remix, adapt, build upon this work noncommercially, and license their derivative works on different terms, provided the original work is properly cited and the use is non-commercial. See: http:// creativecommons.org/licenses/by-nc/4.0/

\section{REFERENCES}

1. American Psychiatric Association (APA). Diagnostic and statistical manual of mental disorders. 5th edn. Arlington, VA: American Psychiatric Publishing, 2013.

2. Williams RJ, Volberg RA, Stevens RMG. The population prevalence of problem gambling: methodological influences, standardized rates, jurisdictional differences, and worldwide trends: report prepared for the Ontario Problem Gambling Research Centre and the Ontario Ministry of Health and Long Term Care. 2012. 
3. Dowling NA, Youssef GJ, Jackson AC, et al. National estimates of Australian gambling prevalence: findings from a dual-frame omnibus survey. Addiction 2016;111:420-35.

4. Gainsbury SM, Russell A, Hing N, et al. The prevalence and determinants of problem gambling in Australia: assessing the impact of interactive gambling and new technologies. Psychol Addict Behav 2014;28:769.

5. Delfabbro P. A review of Australian Gambling Research. Gambling Research Australia, 2008.

6. Dowling NA, Suomi A, Jackson A, et al. Problem gambling and intimate partner violence: a systematic review and meta-analysis. Trauma Violence Abuse 2016;17:43-61.

7. Dowling N, Cowlishaw S, Jackson A, et al. Prevalence of psychiatric co-morbidity in treatment-seeking problem gamblers: a systematic review and meta-analysis. Aust N Z J Psychiatry 2015;49:519-39.

8. Dowling NA, Cowlishaw S, Jackson AC, et al. The prevalence of comorbid personality disorders in treatment-seeking problem gamblers: a systematic review and meta-analysis. J Pers Disord 2015;29:735-54.

9. Cowlishaw S, Merkouris S, Dowling NA, et al. Psychological therapies for pathological and problem gambling. Cochrane Database Syst Rev 2012;11:CD008937.

10. Gooding P, Tarrier N. A systematic review and meta-analysis of cognitive-behavioural interventions to reduce problem gambling: hedging our bets? Behav Res Ther 2009;47:592-607.

11. Yakovenko I, Quigley L, Hemmelgarn BR, et al. The efficacy of motivational interviewing for disordered gambling: systematic review and meta-analysis. Addict Behav 2015;43:72-82

12. Thomas SA, Merkouris SS, Radermacher HL, et al. An Australian guideline for treatment in problem gambling: an abridged outline. Med J Aust 2011;195:664-5.

13. Productivity Commission. Gambling: Productivity Commission inquiry report (Report No. 50). Canberra: 2010.

14. Wilson GT, Zandberg LJ. Cognitive-behavioral guided self-help for eating disorders: effectiveness and scalability. Clin Psychol Rev 2012;32:343-57.

15. Suurvali $\mathrm{H}$, Cordingley J, Hodgins DC, et al. Barriers to seeking help for gambling problems: a review of the empirical literature. J Gambl Stud 2009;25:407-24.

16. Kazdin AE, Blase SL. Rebooting psychotherapy research and practice to reduce the burden of mental illness. Perspect Psychol Sci 2011;6:21-37.

17. Garvin V, Striegel-Moore RH, Wells AM. Participant reactions to a cognitive-behavioral guided self-help program for binge eating: developing criteria for program evaluation. J Psychosom Res 1998;44:407-12.

18. Wagner G, Penelo E, Wanner C. Internet-delivered cognitive-behavioural therapy v. conventional guided self-help for bulimia nervosa: long-term evaluation of a randomised controlled trial. Br J Psychiatry 2013;202:135-41.

19. Rodda S, Lubman DI, Dowling NA, et al. Web-based counseling for problem gambling: exploring motivations and recommendations. J Med Internet Res 2013;15:e99.

20. Rodda SN, Lubman DI, Dowling NA, et al. Reasons for using web-based counselling among family and friends impacted by problem gambling. Asian J Gambling Issues Public Health 2013;3:12.

21. Lal S, Adair CE. E-mental health: a rapid review of the literature. Psychiatr Serv 2014;65:24-32.

22. Lovell K, Bower P, Richards D, et al. Developing guided self-help for depression using the Medical Research Council complex interventions framework: a description of the modelling phase and results of an exploratory randomised controlled trial. BMC Psychiatry 2008;8:1.

23. Andersson $\mathrm{G}$, Carlbring $\mathrm{P}$, Berger $\mathrm{T}$, et al. What makes internet therapy work? Cogn Behav Ther 2009;38:55-60.

24. Cuijpers $P$, Donker T, van Straten A, et al. Is guided self-help as effective as face-to-face psychotherapy for depression and anxiety disorders? A systematic review and meta-analysis of comparative outcome studies. Psychol Med 2010;40:1943-57.

25. Wojtowicz M, Day V, McGrath PJ. Predictors of participant retention in a guided online self-help program for university students: prospective cohort study. J Med Internet Res 2013;15:e96.

26. Coull G, Morris PG. The clinical effectiveness of CBT-based guided self-help interventions for anxiety and depressive disorders: a systematic review. Psychol Med 2011;41:2239-52.

27. Bowman D, Scogin F, Floyd M, et al. Efficacy of self-examination therapy in the treatment of generalized anxiety disorder. J Couns Psychol 1997;44:267.

28. Carter JC, Fairburn CG. Cognitive-behavioral self-help for binge eating disorder: a controlled effectiveness study. J Consult Clin Psychol 1998;66:616-23.
29. den Boer PCAM, Wiersma D, van den Bosch RJ. Why is self-help neglected in the treatment of emotional disorders? A meta-analysis. Psychol Med 2004;34:959-71.

30. Furmark T, Carlbring P, Hedman E, et al. Guided and unguided self-help for social anxiety disorder: randomised controlled trial. Br J Psychiatry 2009;195:440-7.

31. Lidren DM, Watkins PL, Gould RA et al. A comparison of bibliotherapy and group therapy in the treatment of panic disorder. $J$ Consult Clin Psychol 1994;62:865.

32. Nordin S, Carlbring P, Cuijpers $P$, et al. Expanding the limits of bibliotherapy for panic disorder: randomized trial of self-help without support but with a clear deadline. Behav Ther 2010;41:267-76.

33. National Institute for Clinical Excellence (NICE). Core interventions in the treatment and management of anorexia nervosa, bulimia nervosa, and binge eating disorder. London: British Psychological Society, 2004

34. Hirai M, Clum GA. A meta-analytic study of self-help interventions for anxiety problems. Behav Ther 2006;37:99-111.

35. Sanchez-Ortiz VC, Munro C, Stahl D. A randomized controlled tria of internet-based cognitive-behavioural therapy for bulimia nervosa or related disorders in a student population. Psychol Med 2011;41:407-17.

36. Bailer U, de Zwaan M, Leisch F, et al. Guided self-help versus cognitive-behavioral group therapy in the treatment of bulimia nervosa. Int J Eat Disord 2004;35:522-37.

37. Thiels $\mathrm{C}$, Schmidt U, Treasure J, et al. Guided self-change for bulimia nervosa incorporating use of a self-care manual. Am J Psychiatry 1998;155:947-53.

38. Dickerson M, Hinchy J, England SL. Minimal treatments and problem gamblers: a preliminary investigation. J Gambl Stud 1990;6:87-102.

39. Hodgins DC, Currie SR, Currie G, et al. Randomized trial of brief motivational treatments for pathological gamblers: more is not necessarily better. J Consult Clin Psychol 2009;77:950.

40. Hodgins DC, Currie SR, el-Guebaly N. Motivational enhancement and self-help treatments for problem gambling. J Consult Clin Psychol 2001;69:50.

41. Hodgins DC, Currie S, el-Guebaly N, et al. Brief motivational treatment for problem gambling: a 24-month follow-up. Psychol Addict Behav 2004;18:293.

42. Abbott M, Bellringer M, Hodgins D, et al. Effectiveness of problem gambling brief telephone interventions: a randomised controlled trial. Wellington: Ministry of Health, 2012.

43. Petry NM, Ammerman Y, Bohl J, et al. Cognitive-behaviora therapy for pathological gamblers. J Consult Clin Psychol 2006;74:555.

44. LaBrie RA, Peller AJ, LaPlante DA, et al. A brief self-help toolkit intervention for gambling problems: a randomized multisite Trial. Am J Orthopsychiatry 2012;82:278-89.

45. Carlbring $P$, Smit F. Randomized trial of internet-delivered self-help with telephone support for pathological gamblers. J Consult Clin Psychol 2008;76:1090.

46. Casey LM, Oei TPS, Raylu N. Internet-based delivery of cognitive behaviour therapy versus monitoring, feedback and support for pathological gambling. Office of Regulatory Policy, Department of Employment, Economic Development and Innovation, 2009.

47. Hodgins DC, Fick GH, Murray R, et al. Internet-based interventions for disordered gamblers: study protocol for a randomized controlled trial of online self-directed cognitive-behavioural motivational therapy. BMC Public Health 2013;13:10

48. Cunningham JA, Hodgins DC, Bennett $\mathrm{K}$, et al. Online interventions for problem gamblers with and without co-occurring mental health symptoms: protocol for a randomized controlled trial. BMC Public Health 2016;16:624.

49. Patsopoulos NA. A pragmatic view on pragmatic trials. Dialogues Clin Neurosci 2011;13:217-24.

50. Lewin S, Glenton C, Oxman A. Use of qualitative methods alongside randomised controlled trials of complex healthcare interventions: methodological study. BMJ 2009;339:b3496.

51. Chan A-W, Tetzlaff JM, Altman DG, et al. SPIRIT 2013 statement: defining standard protocol items for clinical trials. Ann Intern Med 2013;158:200-7.

52. Treweek S, Zwarenstein M. Making trials matter: pragmatic and explanatory trials and the problem of applicability. Trials 2009;10:37.

53. Andersson G, Estling F, Jakobsson E, et al. Can the patient decide which modules to endorse? An open trial of tailored internet treatment of anxiety disorders. Cogn Behav Ther 2011;40:57-64

54. Kim SW, Grant JE, Potenza MN, et al. The Gambling Symptom Assessment Scale (G-SAS): a reliability and validity study. Psychiatry Res 2009;166:76-84. 
55. Kessler RC, Andrews G, Colpe LJ, et al. Short screening scales to monitor population prevalences and trends in non-specific psychological distress. Psychol Med 2002;32:959-76.

56. Schmidt S, Mühlan H, Power M. The EUROHIS-QOL 8-item index: psychometric results of a cross-cultural field study. Eur J Public Health 2006;16:420-8.

57. Power MJ. Development of a common instrument for quality of life. In: Nosikov A, Gudex C, eds. Developing common instruments for health surveys. Amsterdam: IOS Press, 2003:145-63.

58. Ferris J, Wynne $\mathrm{H}$. The Canadian problem gambling index. Ottawa, ON: Canadian Centre on Substance Abuse, 2001.

59. Holtgraves T. Evaluating the problem gambling severity index. J Gamb/ Stud 2009;25:105-20.

60. Bush K, Kivlahan DR, McDonell MB, et al. The AUDIT alcohol consumption questions (AUDIT-C): an effective brief screening test for problem drinking. Arch Intern Med 1998;158:1789-95.

61. Gordon AJ, Maisto SA, McNeil M, et al. Three questions can detect hazardous drinkers. J Fam Pract 2001;50:313-20.

62. Smith PC, Schmidt SM, Allensworth-Davies D, et al. A single-question screening test for drug use in primary care. Arch Intern Med 2010;170:1155-60.

63. Raylu N, Oei TP. The Gambling Related Cognitions Scale (GRCS): development, confirmatory factor validation and psychometric properties. Addiction 2004;99:757-69.

64. Humke C, Radnitz CL. An instrument for assessing coping with temptation: psychometric properties of the alcohol abuse coping response inventory. Subst Use Misuse 2005;40:37-62.

65. Rodda SN, Lubman DI, lyer R, et al. Subtyping based on readiness and confidence: the identification of help-seeking profiles for gamblers accessing web-based counselling. Addiction 2015;110:494-501.

66. Boudreaux ED, Sullivan A, Abar B, et al. Motivation rulers for smoking cessation: a prospective observational examination of construct and predictive validity. Addict Sci Clin Pract 2012;7:8

67. Hesse M. The Readiness Ruler as a measure of readiness to change poly-drug use in drug abusers. Harm Reduct J 2006;3:1.

68. Soderstrom CA, DiClemente CC, Dischinger PC, et al. A controlled trial of brief intervention versus brief advice for at-risk drinking trauma center patients. J Trauma 2007;62:1102-12; discussion 1111-12.

69. Breslin FC, Sobell LC, Sobell MB, et al. A comparison of a brief and long version of the Situational Confidence Questionnaire. Behav Res Ther 2000;38:1211-20.

70. Ritterband L, Ardalan K, Thorndike F, et al. Real world use of an Internet intervention for pediatric encopresis. J Med Internet Res 2008;10:e16.
71. Thorndike FP, Saylor DK, Bailey ET, et al. Development and perceived utility and impact of an internet intervention for insomnia. E J Appl Psychol 2008;4:32.

72. Tracey TJ, Kokotovic AM. Factor structure of the working alliance inventory. Psychol Assess 1989;1:207-10.

73. Busseri MA, Tyler JD. Interchangeability of the working alliance inventory and working alliance inventory, short form. Psychol Assess 2003;15:193.

74. Miller WR, Brown JM. What I want from treatment. Centre on Alcoholism, Substance Abuse, and Addictions. Albuquerque, NM: 1994

75. Rapee RM, Abbott MJ, Baillie AJ, et al. Treatment of social phobia through pure self-help and therapist-augmented self-help. Br J Psychiatry 2007;191:246-52.

76. Loeb KL, Wilson GT, Gilbert JS, et al. Guided and unguided self-help for binge eating. Behav Res Ther 2000;38:259-72.

77. Titov N, Andrews G, Choi I, et al. Shyness 3: randomized controlled trial of guided versus unguided Internet-based CBT for socia phobia. Aust N Z J Psychiatry 2008;42:1030-40.

78. Holländare F, Johnsson S, Randestad M, et al. Randomized trial of Internet-based relapse prevention for partially remitted depression. Acta Psychiatr Scand 2011;124:285-94

79. Murray E, Khadjesari Z, White I, et al. Methodological challenges in online trials. J Med Internet Res 2009;11:e9.

80. Hernán MA, Hernández-Díaz S. Beyond the intention-to-treat in comparative effectiveness research. Clin Trials 2012;9:48-55.

81. White IR, Kalaitzaki E, Thompson SG. Allowing for missing outcome data and incomplete uptake of randomised interventions, with application to an Internet-based alcohol trial. Stat Med 2011;30:3192-207.

82. Cohen J. Statistical power analysis for the behavioral sciences. 2nd edn. Hillsdale, NJ: L: Erlbaum, 1988.

83. Jacobson NS, Truax P. Clinical significance: a statistical approach to defining meaningful change in psychotherapy research. J Consult Clin Psychol 1991;59:12.

84. Braun V, Clarke V. Using thematic analysis in psychology. Qual Res Psychol 2006;3:77-101.

85. Melville KM, Casey LM, Kavanagh DJ. Dropout from Internet-based treatment for psychological disorders. Br J Clin Psychol 2010;49:455-71.

86. Osman A, Bagge CL, Gutierrez PM, et al. The Suicidal Behaviors Questionnaire-Revised (SBQ-R): validation with clinical and nonclinical samples. Assessment 2001;8:443-54. 